\title{
Aeroelastic Stability of High-Speed Cylindrical Vehicles
}

\author{
Ryan J. Klock ${ }^{*}$ and Carlos E.S. Cesnik ${ }^{\dagger}$ \\ University of Michigan, Ann Arbor, MI 48109-2140
}

\begin{abstract}
Different levels of structural modeling fidelity are evaluated against experimental results for the aeroelastic stability boundaries of an internally pressurized circular cylindrical shell. A modal approach is taken to model the structural dynamics while third-order piston theory is used to model the external and internal surfaces' unsteady aerodynamic pressures. Results are used to inform model improvements of freeflight aero-thermo-elastic simulation in order to accurately predict aeroelastic instabilities in a representative supersonic vehicle. The stability of a finite-element model when inclined to the flow is also considered in an unpressurized condition to inform future work where a cylindrical high-speed vehicle is required to perform maneuvers.
\end{abstract}

\section{Introduction}

The aeroelastic stability of many flight vehicles can be the driving factor in defining their flight envelope. This is typically true for vehicles with a low effective stiffness due to high aspect ratios, slender structures, or high dynamic pressure loading. Ultra high-speed vehicles are no exception and frequently face the added complication of material property degradation resulting from high operating temperatures. Preliminary sizing and performance analysis is carried out numerically for most modern vehicle designs. Yet, the status quo of the computational research upon which these numerical analyses are based largely fall into one of two categories. Either researchers strive to create ever higher-fidelity physics models which are inefficient in a coupled analysis framework or low-fidelity models are used which can be intimately coupled, but do not provide practical solutions due to their simplifying assumptions. Neither of these approaches are optimal for a full design cycle. A middle ground exists between these two in which high to moderate-fidelity models can be closely coupled for the design and analysis of future high-speed vehicles.

Recent work by the authors ${ }^{1-5}$ has been on the development of a set of reduced order models (ROMs) used in the University of Michigan High-Speed Vehicle (UM/HSV) aerothermoelastic simulation framework. These models have focused on the Initial Concept 3.X (IC3X) vehicle ${ }^{6}$ designed by Witeof and Neergaard using the Preliminary Aerothermal Structural Simulation code suite. ${ }^{7}$ The IC3X is a rocket-boosted scramjet vehicle which cruises at Mach 5 and above and at an altitude of 50 thousand feet or higher before entering an unpowered terminal dive. Preliminary simulations of the IC $3 \mathrm{X}$ in the high dynamic pressures of the thermal phase have shown the onset of body flutter similar to the flutter of cylindrical shells in supersonic flow. Figure 1 shows a snapshot of the IC $3 X$ during a time simulation with a deformation pattern characteristic of cylindrical shell flutter. A time trace of the modal amplitudes used to describe the deformation is in Figure 2 and shows exponentially growing oscillation amplitudes typical of flutter.

To evaluate the accuracy of the UM/HSV code in the prediction of this type of flutter and, if necessary, identify areas for improvement, the experimental and analytical work of Olson and Fung ${ }^{8}$ has been considered. In their work, a low-aspect ratio cylindrical copper shell was exposed to supersonic flow ranging from Mach 2.5 to 3.5. The behavior of the shell's flutter motion was studied and the effect of internal pressure and axial load on the flutter boundary was investigated. It was found that the nonlinear geometric behavior of a cylindrical shell induced a series of circumferentially travelling waves whose amplitudes eventually grow at the onset of flutter. Axial load reduced the flutter boundary until the shell buckled, later reproduced by Barr and Stearman ${ }^{9}$ and Bismarck-Nasr. ${ }^{10}$ After buckling, the new corrugated shape was stable. Internal pressure was shown to initially have a destabilizing effect, reducing the flutter boundary, but stabilized the shell at sufficiently high pressures. Early analytical solutions based on shallow shell theory were unable to reconcile this destabilizing and then restabilizing behavior observed in experiments. $^{8}$

\footnotetext{
* Graduate Research Assistant, Department of Aerospace Engineering, Student Member AIAA, rjklock@umich.edu

† Professor, Department of Aerospace Engineering, AIAA Fellow, cesnik@umich.edu 


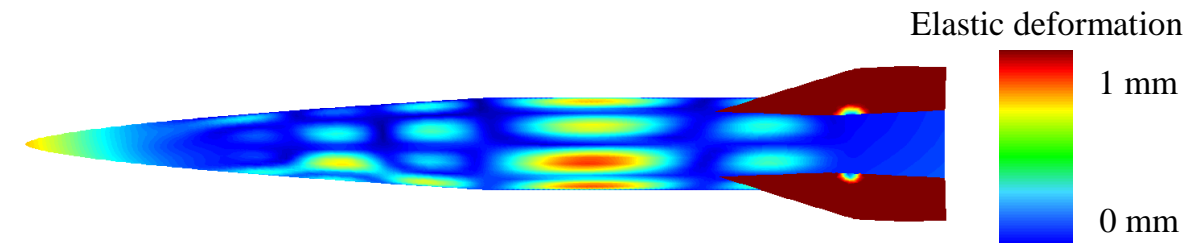

Figure 1: Elastic deformation due to the onset of flutter during aerothermoelastic simulation

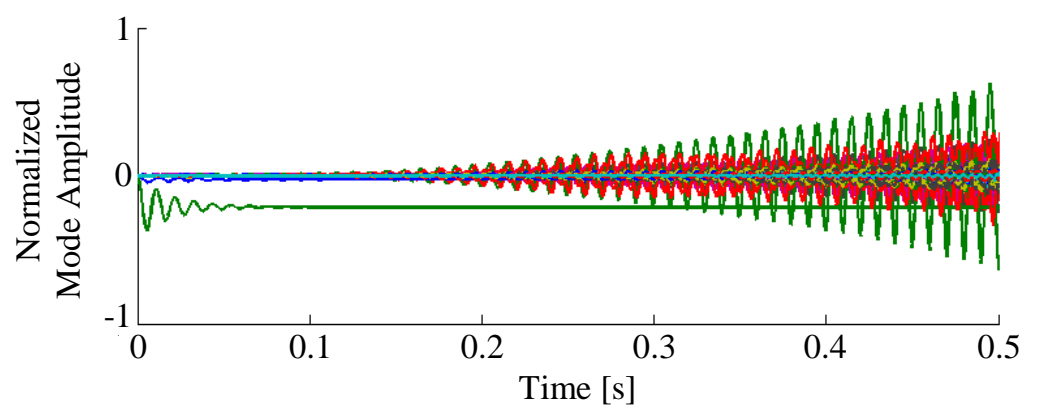

Figure 2: Growth of elastic mode amplitudes in time showing divergent behavior (flutter)

Evensen and Olson ${ }^{11,12}$ later refined the analysis of cylindrical shells using a nonlinear four-mode approach to study the limit cycle oscillation and traveling circumferential waves observed previously. ${ }^{8}$ The works of Barr and Stearman ${ }^{9,13}$ and later Amabili and Pellicano ${ }^{14,15}$ showed how shell imperfections can account for the disagreement between theory and experiment and that the application of nonlinear piston theory did not appear to affect the onset of flutter.

Several works have also studied the application of finite element methods and were summarized by BismarckNasr. ${ }^{16}$ Sabri and Lakis ${ }^{17}$ appears to be the most recent study which focused on the development of a finite element specialized for circular cylindrical shells. Sander's thin shell theory was used to determine displacement fields from exact solutions of the governing equations after which the classical finite element method was applied. The resulting flutter boundary predication was an improvement over past analysis, ${ }^{8}$ but failed to reproduce experimental results.

This paper investigates the applicability of the ROMs previously developed by the authors in the prediction of the flutter of cylindrical shell-type structures and seeks to identify areas that require improvement. This will be done by first attempting to numerically reproduce the flutter experimental results stemming from the work of Olson and Fung ${ }^{8}$ before expanding to consider the stability of the more practical IC $3 \mathrm{X}$ vehicle ${ }^{6}$ case.

\section{Theoretical Development}

To consider the coupled structural dynamics and aerodynamics required to simulate the flutter of a high-speed vehicle or structure, a simulation framework called the University of Michigan High Speed Vehicle (UM/HSV) code was employed. A high-level overview of the framework is summarized and the subset of the UM/HSV methods pertinent to this study is presented here.

\section{A. University of Michigan High Speed Vehicle Framework}

The UM/HSV framework is an in-house coupled-physics code developed over the last several years to consider the interaction of unsteady aerodynamics, aerothermal, thermoelastic, and propulsion models in the simulation of supersonic and hypersonic vehicles over full flight trajectories. It is built upon a collection of both analytical models and ROMs/surrogates that in combination may be used to find vehicle trim solutions, perform stability analyses, and conduct time domain open- and closed-loop simulations. The UM/HSV framework takes advantage of a partitioned approach where substructures are considered with independent subsets of physics models in order to focus fidelity and computational resources on regions of interest. Integration of each partition may be carried out independent of other partitions, at time scales appropriate for the associated processes. Interface equilibriums are then enforced between neighboring partitions, and once a global equilibrium is found, the full vehicle solution is marched forward in time. A high-level outline of this framework is shown in Figure 3. 


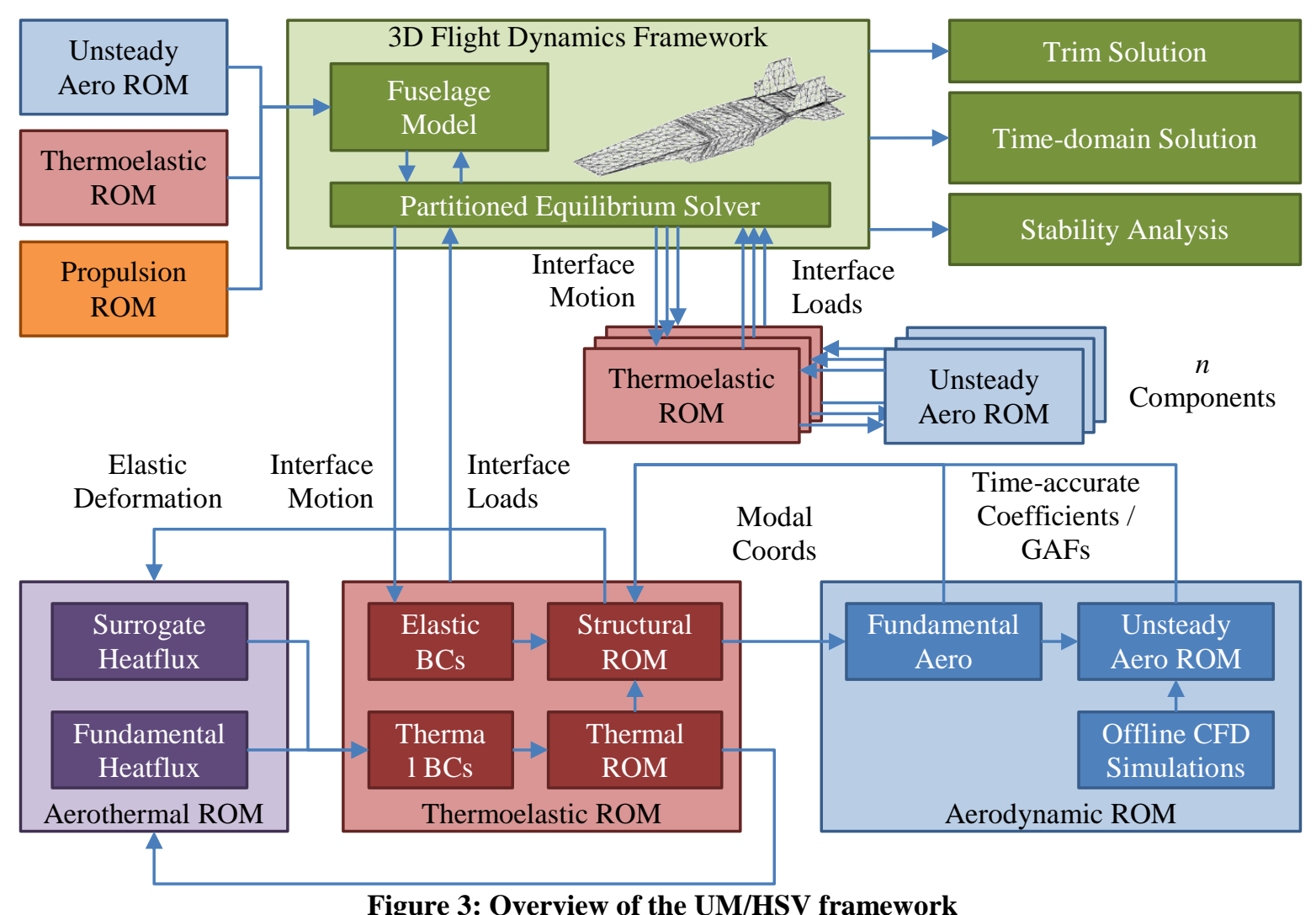

\section{B. Structural Dynamics}

To reduce the order of the structural model, the structural equations of motion may be generalized by a set of orthogonal mode shapes. These shape bases are often composed of free-vibration modes and can be enhanced by including static loading shapes which capture deformation due to specific loading conditions of interest ${ }^{5}$ or higher- $^{-}$ order mode shapes which capture geometric nonlinearities. ${ }^{18-20}$ Consider the undamped full-order structural dynamic equations

$$
M \ddot{x}(t)+K x(t)=F(t),
$$

where $M$ is the mass matrix, $K$ is the stiffness matrix, $F$ is the load vector, $x(t)$ are the physical degrees of freedom, and $t$ is time. The free-vibration mode shapes may be determined from the solution of the eigenproblem

$$
\left(-\omega_{j}^{2} M+K\right) \xi_{j}=0,
$$

where $\omega_{j}$ is the frequency and $\xi_{j}$ is the displacement vector of the $j^{\text {th }}$ mode. Truncation of the shape bases is carried out by excluding modes outside of some frequency range of interest. The physical degrees of are then expressed as a linear combination of these modes such that

$$
x(t)=\left[\begin{array}{ccc}
\xi_{1} & \cdots & \xi_{n} \\
\vdots & \cdots & \vdots
\end{array}\right] d(t)=\Phi d(t),
$$

where $\Phi$ is the basis matrix whose columns are the displacement vectors $\xi$. Substituting (3) into (1) and premultiplying by $\Phi^{T}$ yields

$$
\Phi^{T} M \Phi \ddot{d}(t)+\Phi^{T} K \Phi d(t)=\Phi^{T} F(t),
$$




$$
m \ddot{d}(t)+k_{S} d(t)=f(t)
$$

where the modal terms $m, k$, and $f$ have lower dimension compared to their full-order counterparts.

\section{Unsteady Aerodynamics}

The aerodynamic surface pressures are modeled using $3^{\text {rd }}$ order piston theory ${ }^{21}$

$$
p=p_{\infty}+\rho_{\infty} a_{\infty}^{2}\left\{\left(\frac{w_{n}}{a_{\infty}}\right)+\frac{\gamma+1}{4}\left(\frac{w_{n}}{a_{\infty}}\right)^{2}+\frac{\gamma+1}{12}\left(\frac{w_{n}}{a_{\infty}}\right)^{3}\right\},
$$

where $p$ is pressure, $\rho$ is density, $a$ is the speed of sound, $\gamma$ is the ratio of specific heats, and the subscript $\infty$ denotes far-field values. $w_{n}$ is the velocity that the surface advances into the flow, either due to inclination or unsteady motion normal to the surface face. Both the external and internal pressures are determined based on the external and internal far-field conditions, respectively.

\section{Test Case}

The experimental configuration of Refs. [8,11,12] is considered in this work. A finite element model (FEM) is constructed to match the materials, dimensions, and boundary conditions described in literature. The mode set necessary to construct the experiment within the UM/HSV code is then derived from the FEM and compared to previously published free-vibration mode solutions.

\section{A. Geometry and Properties}

The test article consisted of a circular cylindrical shell formed from electroplated copper with dimensions shown in Figure 4 and whose properties are given in Table 1. The shell section was welded to copper rings on each end which were held to the test mount by $4.763-\mathrm{mm}$ diameter rubber tubing. ${ }^{8}$ Thus the boundaries of the shell were approximately clamped except for axial displacement, which allowed the length of the shell to vary under load. Axial flow was passed over the outer surface while stagnant air was held in a cavity within the shell. Inner surface pressure and freestream flow conditions are summarized in Table 2 .
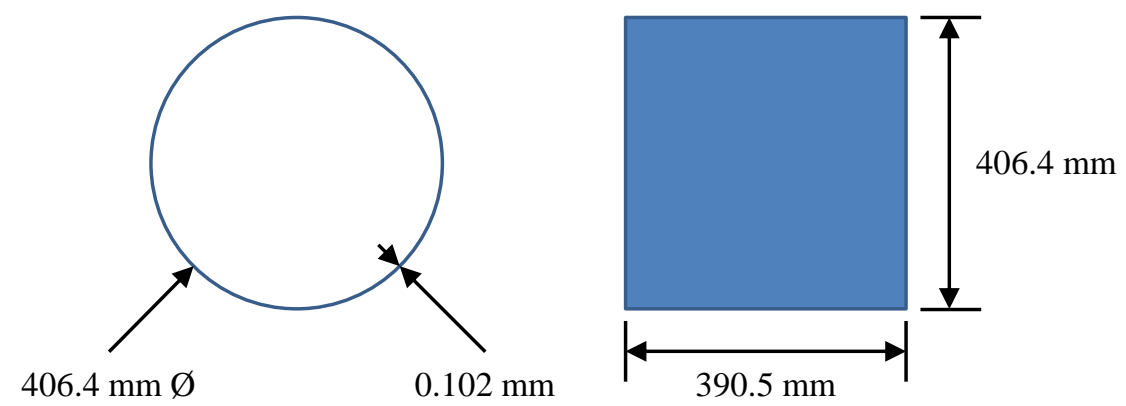

Figure 4: Dimensions of the test cylindrical shell ${ }^{8}$

Table 1: Material properties of copper $^{22,23}$

\begin{tabular}{lcrl} 
Property & Symbol & \multicolumn{2}{c}{ Value } \\
\hline Modulus of elasticity & $E$ & 115 & $\mathrm{GPa}$ \\
Density & $\rho$ & 8960 & $\mathrm{~kg} / \mathrm{m}^{3}$
\end{tabular}


Table 2: Test condition ranges of the cylindrical shell case ${ }^{8}$

\begin{tabular}{lcc} 
Flow condition & Minimum & Maximum \\
\hline Freestream Mach number & 2.5 & 3.5 \\
Freestream total pressure $(\mathrm{kPa})$ & 84.7 & 135.4 \\
Freestream total temperature $(\mathrm{K})$ & 322.0 & 322.0 \\
Inner gauge pressure $(\mathrm{kPa})$ & -0.3 & 27.6
\end{tabular}

\section{B. Finite Element Model}

To numerically recreated the results found by Olson and $\mathrm{Fung}^{8}$, a finite element model was created in the Dassault Abaqus FEM/CAE software ${ }^{24}$ (Figure 5). The model consists of 20,145 nodes and 39,780 S3 linear triangle elements. Loads are applied to the external and internal surfaces based on user-defined subroutines that estimate the surface pressures as described in section II.C using the displacement and displacement rate fields provided by Abaqus. The solution is integrated explicitly in increments no greater than $1 \mu$ s with nonlinear geometry considered. The shell and internal gas are assumed to be at an adiabatic temperature to the external surface flow according to the Eckert's reference temperature method ${ }^{25}$ and are determined at the onset of each time simulation. The throughthickness temperature distribution is assumed to be uniform due to the high thermal conductivity of copper and thinness of the shell wall.

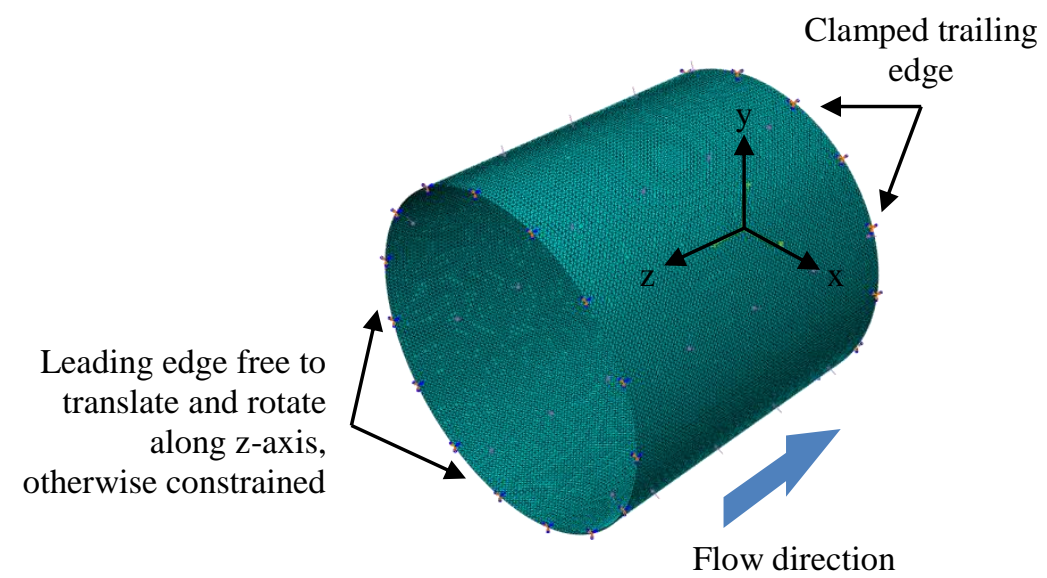

Figure 5: Finite element mesh of the cylinder test case

\section{Cylinder Mode Shapes}

Simulating the cylinder deformation with the UM/HSV code requires the mass-normalized mode shapes. These were determined using the Lanczos solver ${ }^{26}$ included in the Abaqus FEM software for all modes up to $500 \mathrm{~Hz}$ for an unpressurized shell. A selection of mode shapes is shown in Figure 6. A grouping and pattern can be seen in Figure 7 when the mode shapes are organized by numbers of circumferential and longitudinal waves that lie within previously published results, ${ }^{17}$ although differences in the boundary conditions prevented an exact match. The frequency limit was chosen to include all modes experimentally observed by Olson and Fung ${ }^{8}$ to contribute to flutter, namely up to modes with 25 circumferential waves whose frequency is $299 \mathrm{~Hz}$. Adding internal pressure causes the cylinder to inflate and increases the membrane stress, stiffening the structure. To account for this in the $\mathrm{UM} / \mathrm{HSV}$ code, multiple sets of mode shapes and frequencies were determined for $0,1,2,3,4,5,6$, and $7 \mathrm{kPa}$ of internal gauge pressure. The variation of the mode frequencies is shown in Figure 8. 

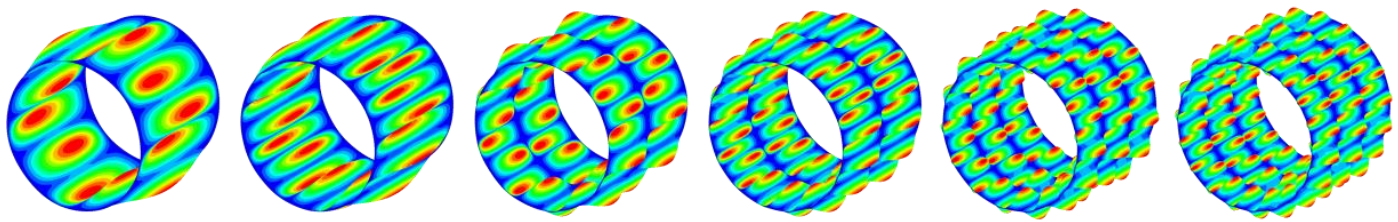

Displacement
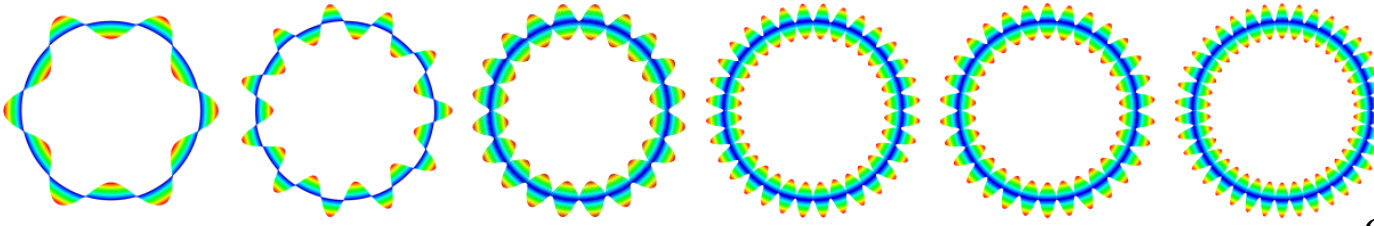

6

11

10

16

15

2

3

19 waves

3 Longitudinal half-waves

Figure 6: Sample unpressurized mode shapes
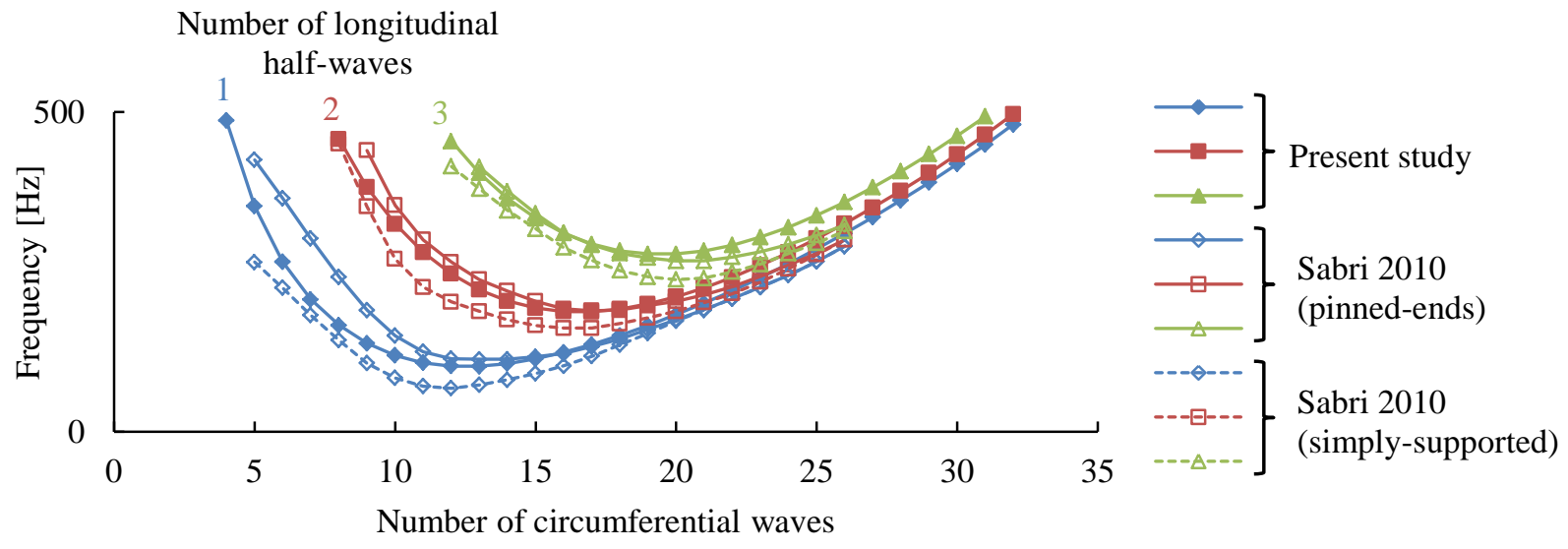

Figure 7: Unpressurized mode frequencies

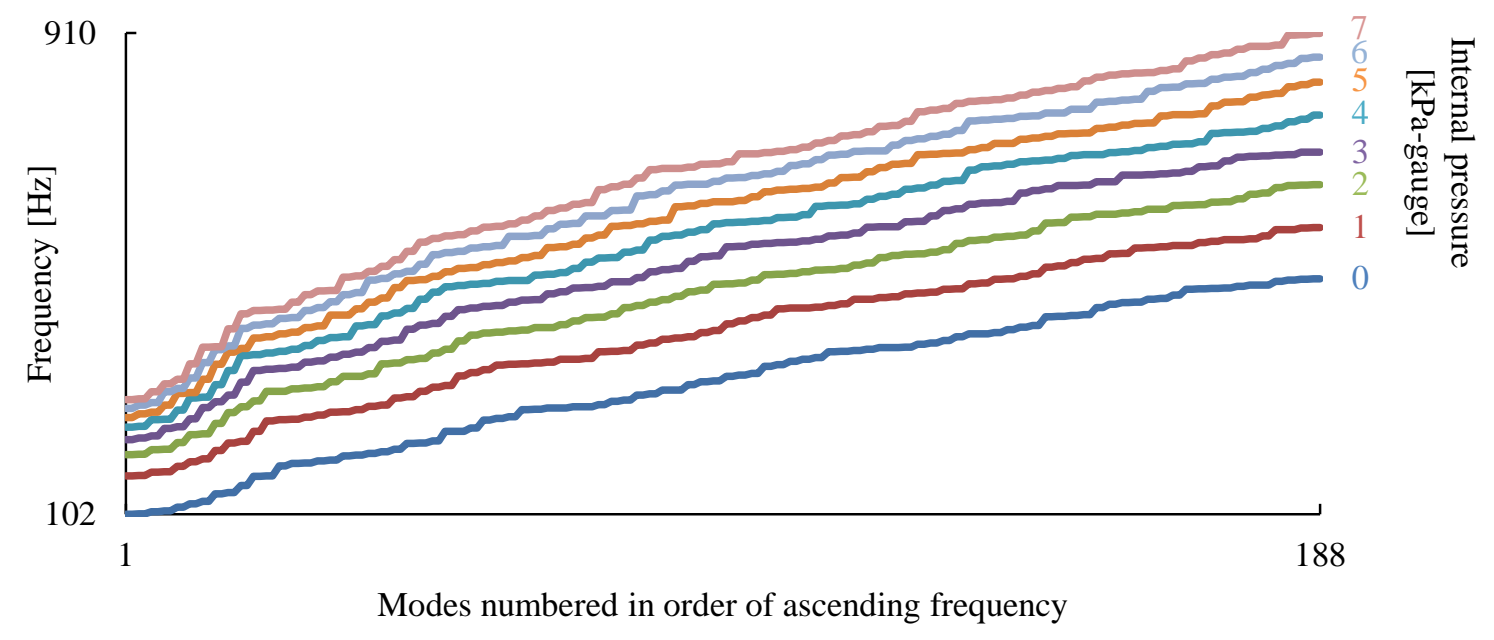

Figure 8: Variation of mode frequency due to internal pressure 


\section{Results and Discussion}

Aeroelastic simulations were conducted for the FEM and UM/HSV models and used to determine the stability boundaries with various amounts of internal gauge pressure. The results are compared with published experimental, analytical, ${ }^{8}$ and numerical ${ }^{17}$ results. The FEM model is then inclined to the flow in order to determine the effect of the angle of attack on the stability boundary.

\section{A. Effects of Internal Pressure on Flutter}

The onset of flutter was observed to be the limiting factor in stability when the model was exposed to flow aligned with its axis, $0^{\circ}$ angle of attack. Snapshots of a simulation exhibiting flutter are shown in Figure 9 along with a trace of the radial displacement of a node midway along the upper surface. For each simulation, the cylinder was started in an unrelaxed state, meaning that no static solution was found which arose from the difference of internal and external pressures. Thus, a perturbation was induced at the start of each simulation due to the instantaneous application of differential pressure between the inside and outside of the cylinder. This pressure step would cause an initial displacement of a maximum of approximately $30 \mu \mathrm{m}$. Cases which exhibited flutter all appeared to follow a similar evolution which started with large, low frequency waves which traveled axially from the leading to trailing edge. Interference of these waves and their reflections from the boundaries would yield increasingly smaller waves, some of which traveled circumferentially. These small, circumferential waves would grow in amplitude until the end of the simulation.

Displacement traces at the surface location shown in Figure 9 were extracted from the FEA solutions. The traces are divided into 0.02-s long windows in which the maximum absolute displacements were determined. If the maximum displacement in each window grew over time for a given case, then that case was considered unstable. The window length was selected such that the lowest frequency natural mode determined in section III.C would complete at least one cycle per window. The boundary between stable and unstable pressure conditions was then converged upon for several internal pressure cases by varying the freestream static pressure for Mach 3 flow with a total temperature of $322 \mathrm{~K}$. The results are shown in Figure 10.

Overall, the predicted flutter boundary from this study compares better with the experimental results of Olson and Fung ${ }^{8}$ than either the analytical results of Olson and Fung ${ }^{8}$ or FEA results of Sabri and Lakis. ${ }^{17}$ Due to the uncertainties in the numerical convergence tolerance in the flutter identification process, the present study FEA results are augmented by a series of error bars to capture that uncertainty. The destabilizing effect of moderate amounts of internal gauge pressure between 1 and $4 \mathrm{kPa}$ is captured before recovering above $5 \mathrm{kPa}$. However, the FEA results under estimate the experimental result for most cases.

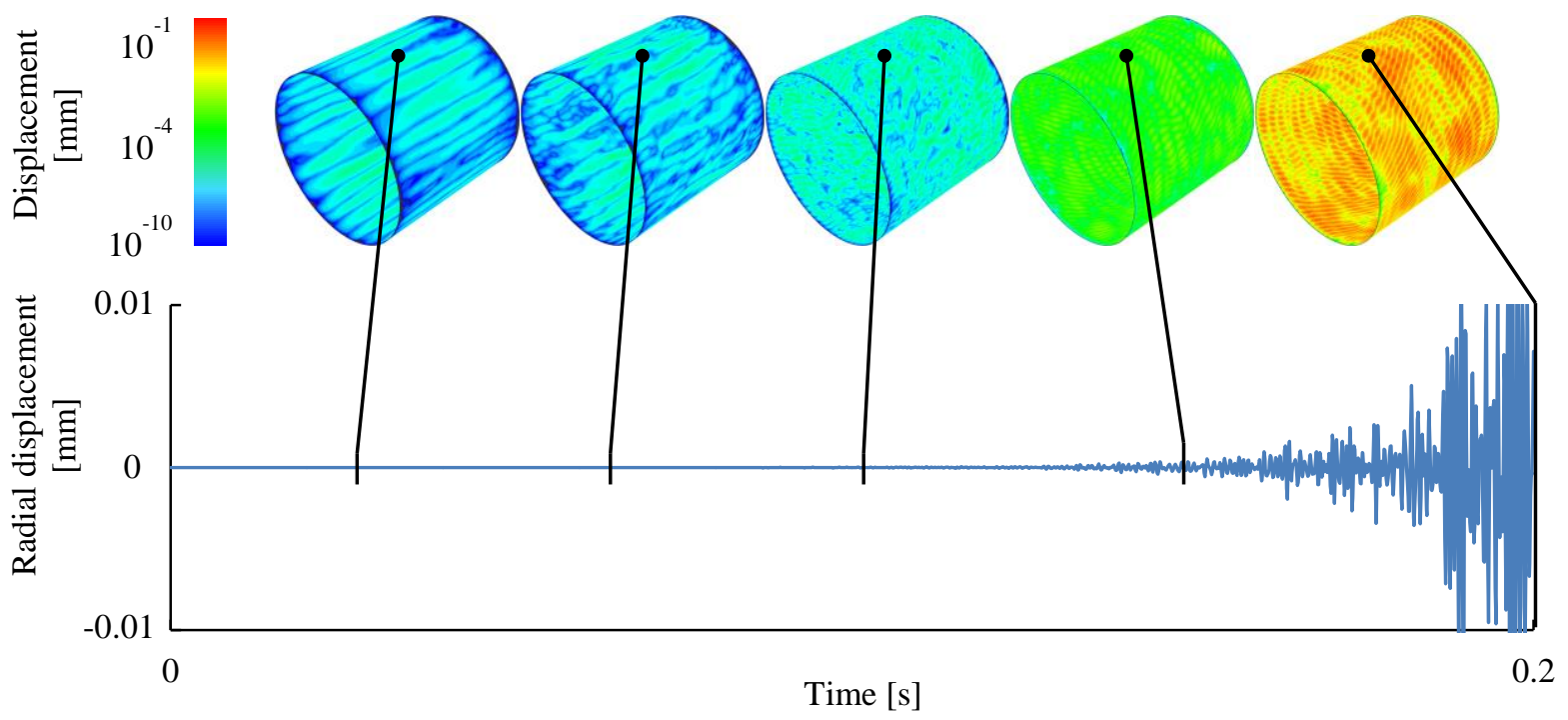

Figure 9: Sample snapshots and trace of displacement for a simulation exhibiting flutter, $4.8 \mathrm{kPa}$ freestream pressure, $0.0 \mathrm{kPa}$-gauge internal pressure, $0^{\circ}$ angle of attack, Mach 3 flow 


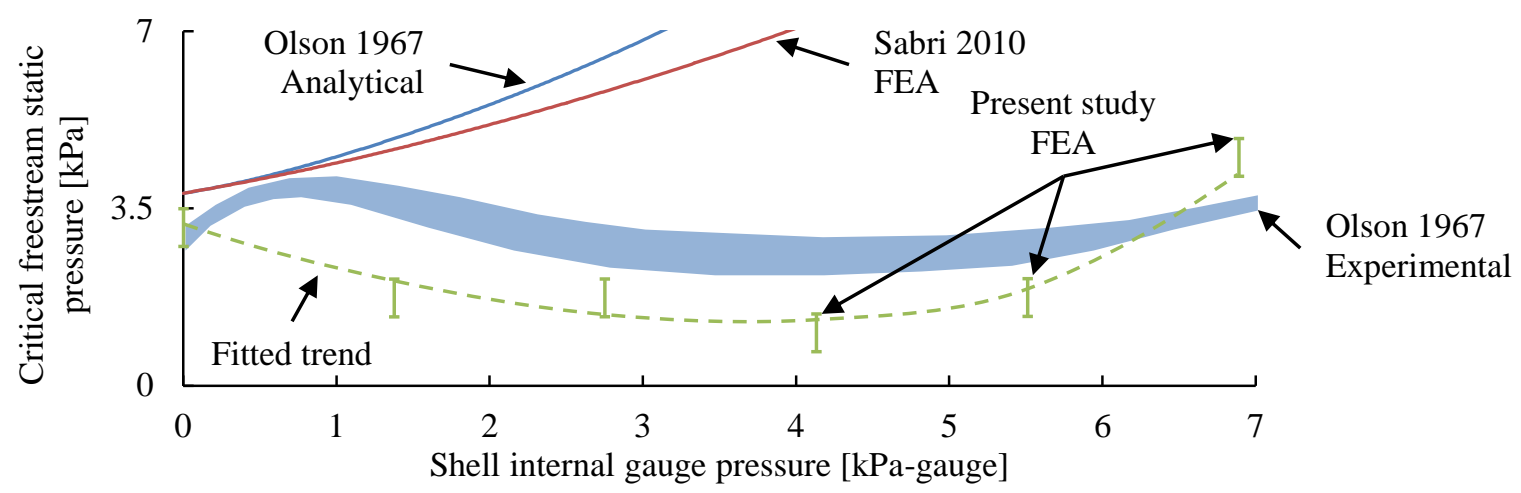

Figure 10: Experimental flutter boundary of the cylinder at Mach 3, $322 \mathrm{~K}$ total temperature, $0^{\circ}$ angle of attack. Numerical flutter boundary predictions from present and previous studies ${ }^{8,17}$ are included for comparison

From observing the displacement field, this destabilization and then re-stabilization appeared to arise from two competing effects:

1. As the internal gauge pressure was increased, the shell was pushed outward, into the flow. Most of the shell expanded uniformly except near the leading and trailing edges where the boundary conditions restrained the cross section radius. A ramp was created which resulted in a region of slightly higher pressure sufficient to initiate oscillations in the shell near the leading edge (Figure 11).

2. Increasing the internal pressure also induced a geometric stiffening of the structure by placing the shell in tension. If the pressure was sufficiently increased, then this stiffening overcame the previous destabilizing effect.

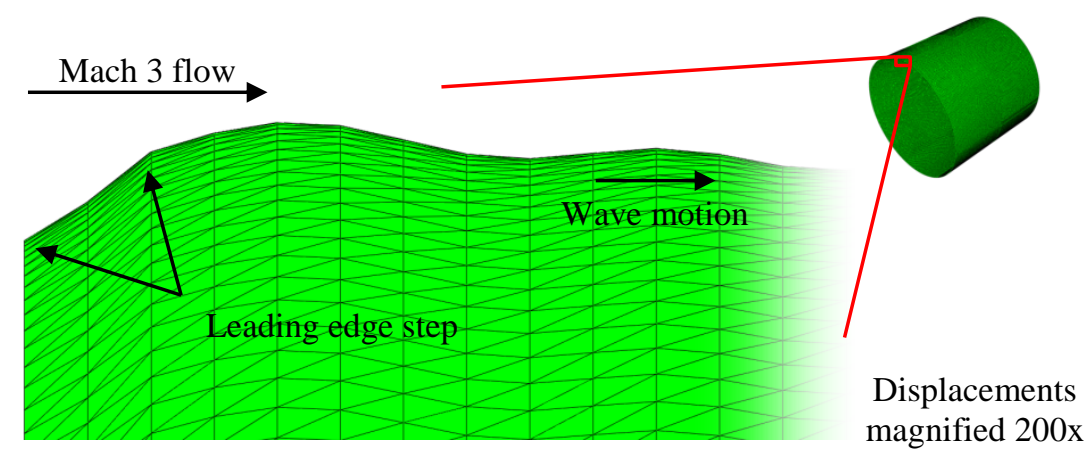

Figure 11: Example of the development of oscillations near leading edge step due to internal pressurization

\section{B. Effects of Internal Pressure on UM/HSV Model Flutter}

With an FEA model which could capture the destabilizing effects of moderate internal pressure to compare with, the cylinder model was constructed with the UM/HSV code. Aeroelastic simulations were conducted using the modes and frequencies determined in section III.C. Snapshots of a simulation that exhibited flutter are shown in Figure 12.

Despite simulating the same flow conditions as used in Figure 9, it can be seen from Figure 12 that the process of flutter onset is somewhat different when considered by the UM/HSV code. Little initial displacement is observed and the growth of the oscillations that do appear is more sudden than in the FEM case. The modal approach taken in this case also results in the appearance of much more ordered displacement patterns on the shell, despite the large number of mode shapes considered. Circumferentially travelling waves appear early, without an initial transient period. The flutter boundary of the cylinder model in the UM/HSV code was converged upon using the same technique as with the FEM model. The resulting boundary is compared to previous results in Figure 13.

The UM/HSV code was able to predict the correct magnitude of the flutter boundary but did not capture the destabilizing effect of internal pressure. Instead it predicted a monotonic increase in the flutter boundary similar to previous works ${ }^{8,10,14,17}$ but shifted toward the experimental boundary. During early simulations, it was observed that the UM/HSV solution was unable to represent the outward expansion of the cylinder due to an internal gauge 
pressure as the free-vibration mode shape which would be used to represent this deformation was well outside of the $500 \mathrm{~Hz}$ limit imposed by the authors for the unpressurized case. To overcome this limitation, the mode set was enhanced by including the deformation of a static solution of the cylinder subjected to the steady component of the internal pressure considered for each case. The modified Gram-Schmidt algorithm was used to orthogonalize the statically loaded shape against the free-vibration derived bases and was appended to the bases set. ${ }^{5}$

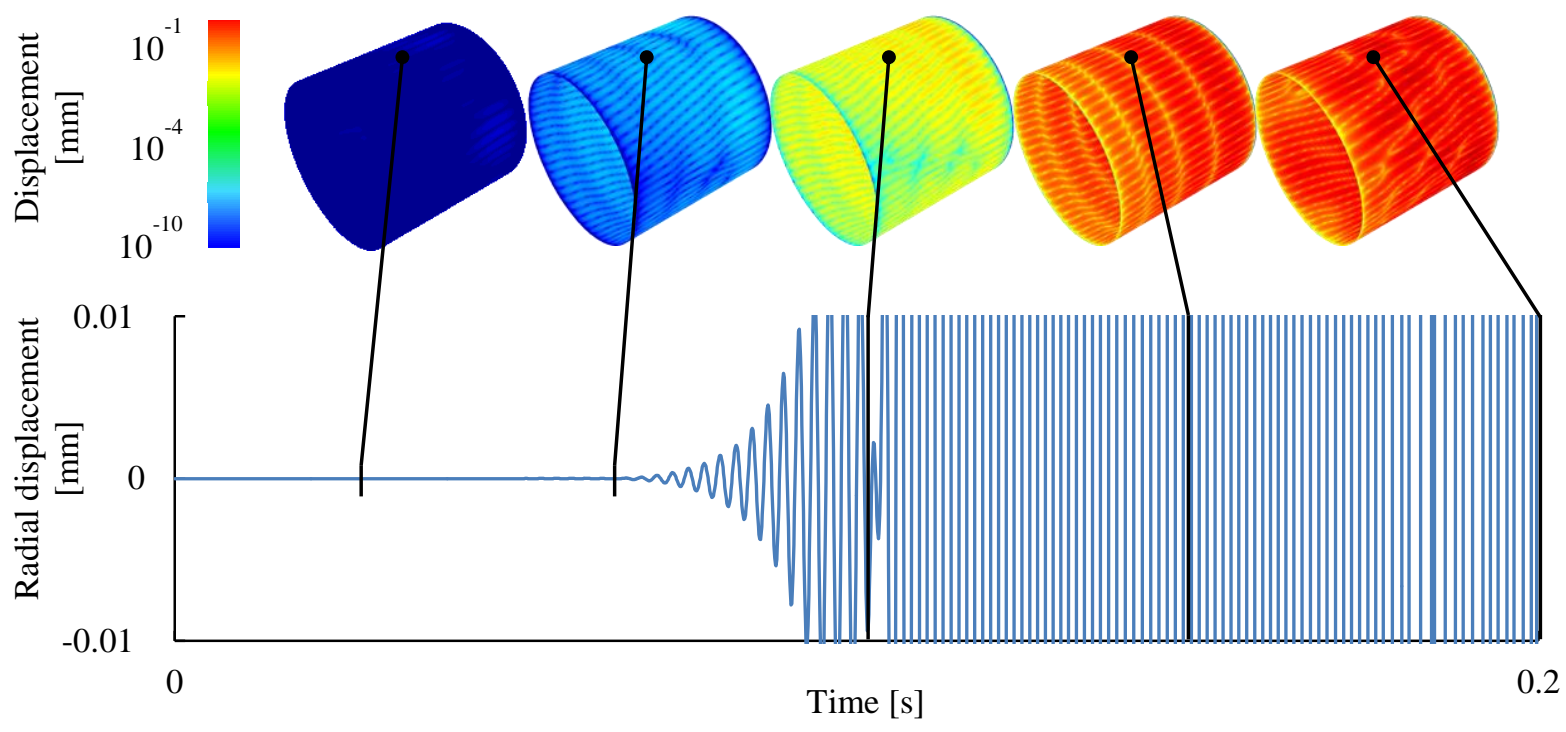

Figure 12: Sample snapshots and trace of displacement for a simulation exhibiting flutter as processed by the $\mathrm{UM} / \mathrm{HSV}$ code, $4.8 \mathrm{kPa}$ freestream pressure, $0.0 \mathrm{kPa}$-gauge internal pressure, $0^{\circ}$ angle of attack, Mach 3 flow

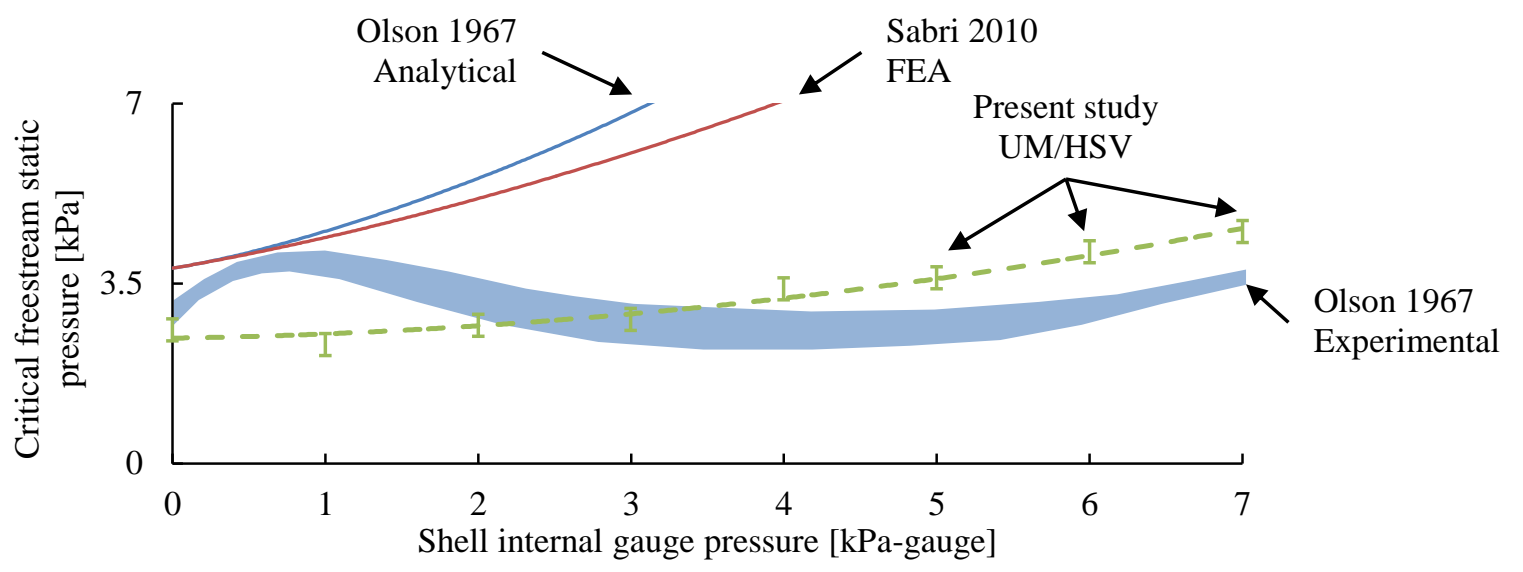

Figure 13: Comparison of Mach 3, $322 \mathrm{~K}$ total temperature flutter boundary for the UM/HSV model with previous analytical, ${ }^{8}$ numerical, ${ }^{17}$ and experimental results ${ }^{8}$

\section{Effect of Angle of Attack on Stability Boundary}

As the eventual goal of this work is to improve the aeroelastic stability predictions of the UM/HSV code for a maneuvering high-speed vehicle, it was of interest to study the effect of the angle of attack on the stability of a cylindrical shell. To do this, the cylinder FEM was incrementally inclined up to $20^{\circ}$ to the Mach 3 free stream flow. The limit of $20^{\circ}$ was selected since at this angle, the windward surface normal Mach number is approximately 1 for the undeformed cylinder. This represented the upper limit of inclination due to the isentropic assumptions of the piston theory ${ }^{21}$ aerodynamic model considered in this work.

Unlike with axial flow, instability of the cases with inclined flow often resulted in the buckling of the cylinder shell on the windward side, similar to the case shown in Figure 14. Depending on the exact manner in which the shell buckled, the post-buckled shape could either be stable or could continue to exhibit low frequency oscillations on the sides on the order of $20 \mathrm{~Hz}$. The stability boundary was converged upon as described previously and is shown 
in Figure 15. Error bars were used to represent the tolerance of the convergence process. Increased angles of attack lead to a decreased stability boundary as low as $0.18-\mathrm{kPa}$ freestream pressure at $20^{\circ}$. This represented a $94 \%$ loss in the critical freestream flutter pressure.

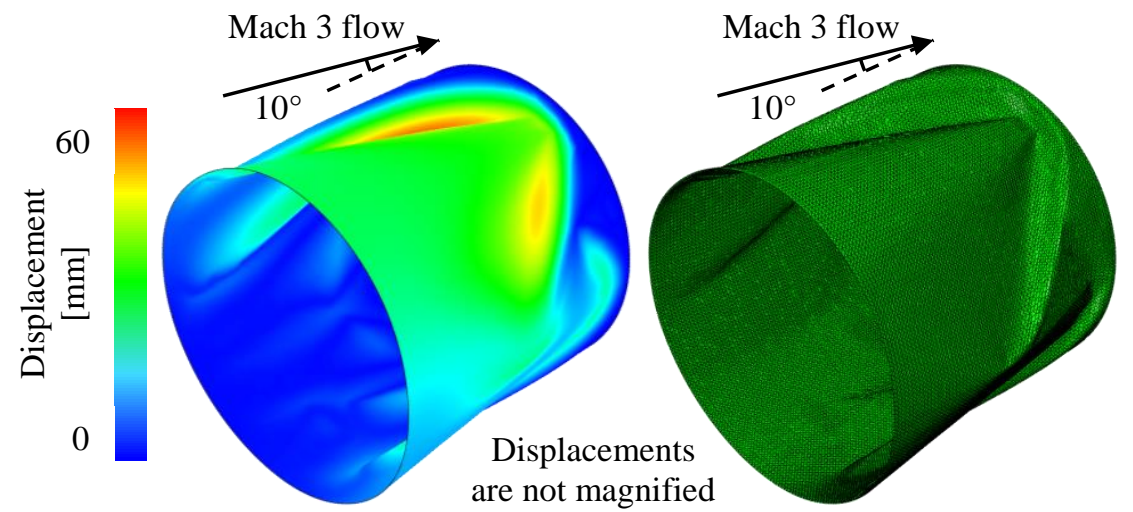

Figure 14: Example of buckled cylinder due to inclined flow, $0.69 \mathrm{kPa}$ freestream pressure, 0 kPa-gauge internal pressure, Mach 3 flow, $10^{\circ}$ angle of attack

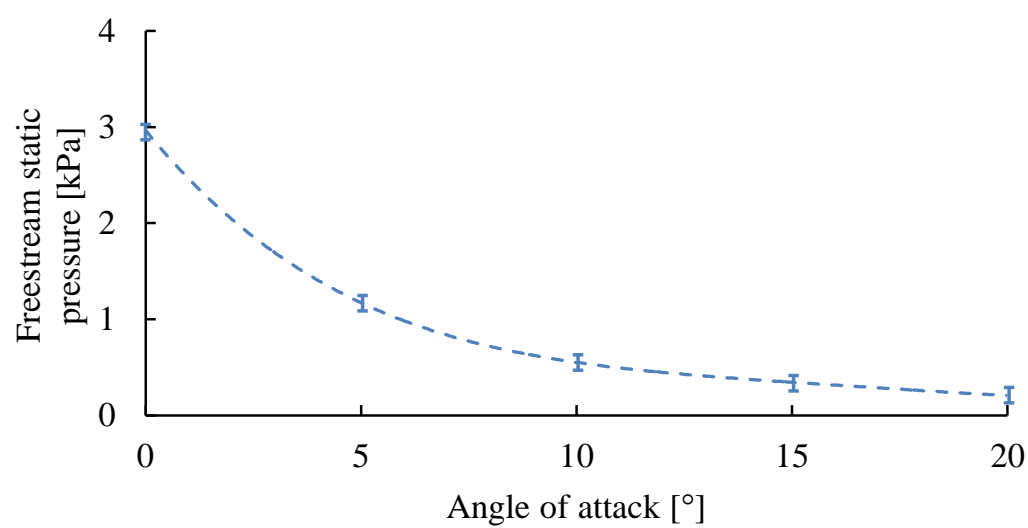

Figure 15: Stability boundary of the cylinder FEM at an angle of attack, 0 kPa-gauge internal pressure, Mach 3 flow

\section{Conclusions}

The cylindrical shell flutter in supersonic flow was revisited. A nonlinear FEM was constructed which was able to capture the destabilizing effects of moderate amounts of internal pressure and circumferentially traveling waves on the aeroelastic stability of a circular cylindrical shell exposed to axial flow at Mach 3, observed experimentally by Evensen, Olson, and Fung. ${ }^{8,11,12}$ While not an exact match to experiment, the results where an improvement over previous modern FEM solutions presented by Sabri and Lakis. ${ }^{17}$ Free-vibration modes up to $500 \mathrm{~Hz}$ were derived from the nonlinear FEM in an unpressurized condition and the corresponding frequencies were shown to match well with previously published results. These mode shapes, as well as their pressurized counterparts up to $7 \mathrm{kPa}$-gauge, were then used as a structural deformation basis for the UM/HSV code.

It was shown that the UM/HSV code was able to approximately match the experimental results and but predicted a monotonically increasing stability boundary due to shell pressurization. Comparison of the displacement fields at the onset of flutter between the FEM and UM/HSV models showed that the axisymmetric expansion of the shell due to internal pressure appeared to destabilize the shell by inclining the surface near the leading edge. This inclination resulted in a slightly higher local pressure which would induce oscillation but was not captured by in the UM/HSV solution despite the inclusion of mode shapes derived from the static solutions of the cylinder inflation.

In preparation for future work, the unpressurized shell was also placed at an angle of attack to a Mach 3 flow. Applying an angle of attack was shown to decrease the critical freestream pressure by as much as $94 \%$ at $20^{\circ}$ and often lead to buckling of the shell. The post-buckled shells were then stable with minor oscillations until the end of each simulation. 


\section{Acknowledgments}

This work was sponsored by the Air Force Research Laboratory (AFRL), Munitions Directorate, Eglin Air Force Base, Florida. The technical monitors are Dr. Crystal Pasiliao and Dr. Daniel Reasor. Opinions, interpretations, conclusions, and recommendations are those of the authors and are not necessarily endorsed by the United States Government.

\section{References}

$\checkmark 1 \quad$ Falkiewicz, N. J. and Cesnik, C. E. S., "Proper Orthogonal Decomposition for Reduced-Order Thermal Solution in Hypersonic Aerothermoelastic Simulations," AIAA Journal, Vol. 49, No. 5, May 2011, pp. 9941009.

2 Klock, R. J. and Cesnik, C. E. S., "Aerothermoelastic Simulation of Air-Breathing Hypersonic Vehicles," Proceedings of the 55 $5^{\text {th }}$ AIAA/ASME/ASCE/AHS/ASC Structures, Structural Dynamics, and Materials Conference, National Harbor, Maryland, 13-17 January 2014, AIAA 2014-0149.

-3 Klock, R. J., and Cesnik, C. E. S., "Aerothermoelastic Reduced-Order Model of a Hypersonic Vehicle," Proceedings of the AIAA Atmospheric Flight Mechanics Conference, Dallas, Texas, 22-26 June 2015, AIAA 2015-2711.

$4 \quad$ Klock, R. J. and Cesnik, C. E. S., "Nonlinear Thermal Reduced Order Models of a Hypersonic Vehicle," Proceedings of the $15^{\text {th }}$ Dynamics Specialists Conference, San Diego, California, 4-8 January 2016, AIAA 2016-1322.

-5 Falkiewicz, N. J. and Cesnik, C. E. S., "Enhanced Modal Solutions for Structural Dynamics in Aerothermoelastic Analysis," Journal of Aircraft, published online 11 November 2016.

6 Witeof, Z. D. and Neergaard, L. J., "Initial Concept 3.0 Finite Element Model Definition," Air Force Research Laboratory, Rept. AFRL-RWWV-TN-2014-0013, Eglin AFB, Florida, April 2014 (96ABW2014-0141).

7 Pasiliao, C. L., Sytsma, M. J., Neergaard, L. J., Witeof, Z. D., and James.W., T., "Preliminary Aerothermal Structural Simulation," Proceedings of the 14th AIAA Aviation Technology, Integration, and Operations Conference, Atlanta, Georgia, 16-20 June 2014, AIAA 2014-2292.

-8 Olson, M. D. and Fung, Y. C., "Comparing Theory and Experiment for the Supersonic flutter of Circular Cylindrical Shells," AIAA Journal, Vol. 5, No. 10, October 1967, pp. 1849-1856.

-9 Barr, G. W. and Stearman, R. O., "Influence of a Supersonic Flowfield on the Elastic Stability of Cylindrical Shells," AIAA Journal, Vol. 8, No. 6, June 1970, pp. 993-1000.

-10 Bismarck-Nasr, M. N., "Finite Element Method Applied to the Supersonic Flutter of Circular Cylindrical Shells," International Journal for Numerical Methods in Engineering, Vol. 10, 1976, pp. 423-435.

11 Evensen, D. A. and Olson, M. D., "Nonlinear Flutter of a Circular Cylindrical Shell in Supersonic Flow," NASA TN-D-4265, December 1967.

-12 Evensen, D. A. and Olson, M. D., "Circumferentially Traveling Wave Flutter of a Circular Cylindrical Shell," AIAA Journal, Vol. 6, No. 8, August 1968, pp. 1522-1527.

-13 Barr, G. W. and Stearman, R. O., "Aeroelastic Stability Characteristics of Cylindrical Shells Considering Imperfections and Edge Constraint," AIAA Journal, Vol. 7, No. 5, May 1969, pp. 912-919.

-14 Amabili, M. and Pellicano, F., "Nonlinear Supersonic Flutter of Circular Cylindrical Shells," AIAA Journal, Vol. 39, No. 4, April 2001, pp. 564-573.

-15 Amabili, M. and Pellicano, F., "Multimode Approach to Nonlinear Supersonic Flutter of Imperfect Circular Cylindrical Shells," Journal of Applied Mechanics, Vol. 69, March 2002, pp. 117-129.

-16 Bismarck-Nasr, M. N., "Finite Elements in Aeroelasticity of Plates and Shells," Applied Mechanics Review, Vol. 49, No. 10, October 1996, pp. S17-S24.

-17 Sabri, F. and Lakis, A. A., "Finite Element Method Applied to Supersonic Flutter of Circular Cylindrical Shells," AIAA Journal, Vol. 48, No. 1, January 2010, pp. 73-81.

18 Ritter, M. and Cesnik, C. E. S., "An Enhanced Modal Approach for Large Deformation Modeling of WingLike Structures," Proceedings of the 56 $6^{\text {th }}$ AIAA/ASCE/AHS/ASC Structures, Structural Dynamics, and Materials Conference, Kissimmee, Florida, 5-9 January 2015, AIAA 2015-0176.

19 Allen, M. S., Kuether, R. J., and Deaner, B., "A Numerical Continuation Method to Compute Nonlinear Normal Modes Using Modal Reduction," Proceedings of the 53 ${ }^{\text {rd }}$ AIAA/ASME/ASCE/AHS/ASC Structures, Structural Dynamics and Materials Conference, Honolulu, Hawaii, 23-26 April 2012, AIAA 2012-1970.

20 Kuether, R. J. and Allen, M. S., "Nonlinear Modal Substructuring of Systems with Geometric Nonlinearities," Proceedings of the 54th AIAA/ASME/ASCE/AHS/ASC Structures, Structural Dynamics, and 
Materials Conference, Boston, Massachusetts, 8-11 April 2013, AIAA 2013-1521.

-21 Holt, A. and Garabed, Z., "Piston Theory - A New Aerodynamic Tool for the Aeroelastician," Journal of the Aeronautical Sciences, Vol. 23, No. 12, December 1956, pp. 1109-1118.

22 Anon., "Young Modulus of Elasticity for Metals and Alloys," The Engineering Toolbox [online database], http://www.engineeringtoolbox.com/young-modulus-d_773.html [retrieved 19 June 2016].

-23 White, G. K. and Collocott, S. J., "Heat Capacity of Reference Materials: Cu and W," Journal of Physics, Chemistry, and Reference Data, Vol. 13, No. 4 1984, pp. 1251-1257.

24 Abaqus, “Abaqus 6.13 Documentation,” Dassault Systèmes, Providence, Rhode Island, 2013.

25 Eckert, E. R. G., "Survey of Boundary Layer Heat Transfer at High Velocities and High Temperatures," WADC Technical Report 59-624, June 1960.

-26 Ojalvo, I. U. and Newman, M., "Vibration Modes of Large Structures by an Automatic Matrix-Reduction Method," AIAA Journal, Vol. 8, No. 7, July 1970, pp. 1234-1239. 
This article has been cited by:

1. Joseph Schoneman, Christopher Ostoich, Lucas Jarman, Chris I. VanDamme, Matthew Allen. Impact of Flow and Structural Nonlinearities on Hypersonic Panel Flutter Predictions . [Citation] [PDF] [PDF Plus] 